\title{
Management of keratoplasty in the early stages
}

\author{
ALBERTO URRETS-ZAVALIA \\ From the Ophthalmic Clinic, Córdoba National University, Córdoba, Argentina
}

SUMmARY Frequent, if not daily, slit-lamp examination of the patient with a penetrating transplant is indicated for more than one reason: it will lead to the early detection of any defect of the hostgraft junction or of the graft's posterior surface; it will permit an early diagnosis of the peripheral cornea-iris touch syndrome with all its potential consequences (acute, irreversible mydriasis, iris atrophy, and now and then secondary glaucoma); it will give an exact clue to when to remove the continuous sutures long after these have been covered by epithelium; and it will alert the surgeon in the event of a localised infection. Early and late opacification of the graft will also be correctly appraised, so that the surgeon will know when to wait and when to take the appropriate action.

Despite the development of new methods for the preservation of corneal donor material, in most centres where corneal transplantation is performed routinely fresh material is preferred. The donor eyes are removed less than six hours after death and are stored in a moist chamber at $4^{\circ} \mathrm{C}$ for a maximum of 24 hours. As a rule only material from donors aged 50 years or less is used so as to make sure that the grafted endothelium will be of optimal viability.

Failures due to damage inflicted to initially good material have become rare since proper microsurgical techniques and tools have been used. The main causes of failure that remain are, first, those inherent in some conditions in which surgery has a guarded prognosis, and, secondly, several complications whose appearance seems unrelated to the quality of the transplanted disc, to the instrumentation employed, or to the surgeon's dexterity.

This paper is not intended to be a list of the pitfalls encountered during or after the performance of partial penetrating keratoplasty, as this would be futile. Its aim is to study some early prognostic signs as they become apparent in the postoperative course so that adequate and timely treatment may be instituted.

\section{Graft defects}

A point which has never been stressed sufficiently is that even in the case of an avascular and uninfected host cornea, the impeccable transplantation of healthy tissue which has been carefully examined preoperatively may not always result at once in a

Address for reprints: Prof A. Urrets-Zavalia, Casilla de Correo 301, 5000 Córdoba, Argentina. crystal-clear graft, or in a graft that can be considered wholly satisfactory. While some of the deficiencies detected early after the operation require no treatment and will mend spontaneously, others are a bad omen and require that immediate measures be taken if success is to be attained.

A crinkled posterior surface of the graft is a frequent finding after penetrating keratoplasty. Owing to the presence of folds in Descemet's membrane it may be caused either by stromal oedema or by an uneven tension of the direct or running monofilament sutures which keep the graft in place. If the donor cornea is waterlogged when the graft is cut, the imbibition tends to disappear once the graft is accurately applied. Initially increased, the corneal thickness will return to normal in a few days' time. When the wrinkles in Descemet's membrane result from uneven traction, they will persist until the sutures are removed and will not be accompanied by an increased graft thickness.

If a single or double continuous suture is used, either alone or in combination with a number of border-to-border sutures, apposition of the wound edges is good. Because of the very fact that the edges are fitted together quite closely, some marginal epithelial oedema develops and may remain visible for weeks. The trouble with this is that it will cause an irregularity of the corneal reflex, as may be seen with a keratoscopic disc, and render any ophthalmometric reading useless or grossly lacking in precision.

There are cases in which the graft is clear when it is placed in position only to become oedematous later. This may occur even if proper suturing has been done. An opalescence, slight at first, may 
subsequently take on the appearance of a striate keratitis, which indicates unequivocally that the endothelium is suffering or, more rarely, that there is a defective alignment between the graft and the host, so that the aqueous seeps into the stroma. Not uncommonly some degree of epithelial oedema is seen in large parts of the graft. The degree of the opacity which may develop in the graft after surgery varies. It may be very faint, merely a slight haze, discernible only with retroillumination, or it may be rather dense. It may affect the entire cornea or only a sector. And it may lie in the endothelium, the stroma, or the epithelium. Such an appearance causes grave concern to the surgeon although it may produce no permanent damage and vanish without trace in a couple of weeks. The grafted cornea then regains its normal thickness unless endothelial disease existed in the donor cornea.

In those cases in which the cornea is still imperfectly transparent at the end of two weeks a subTenon's injection of a slow-resorption steroid is indicated. This may be done as an office procedure after the removal of the direct sutures. Far from retarding endothelial recovery (Sánchez and Polack, 1974), the administration of topical steroids even in large doses seems to have a highly beneficial effect in any situation where the endothelium is less than perfectly healthy. But if the graft does not clear in a very few more days it must be replaced, as the chances of its becoming clear again are slim (Barraquer and Rutllán, 1969).

When oedema and opacification of the graft become manifest at about the third postoperative week, the conclusion should be that one is faced with an allograft reaction, a problem which falls beyond the scope of this paper. In this respect let us only say that rejection may be preceded by an increased graft thickness, which becomes apparent before the development of any loss in transparency when serial measurements with the Haag-Streit pachometer are made. Another early sign of rejection is represented by the deposition of a number of small grey precipitates on the posterior surface of the graft.

Both in the case of the early opacification due to the poor quality of the donor material and in that of an incipient true allograft reaction, direct examination of the endothelium with a specular reflection microscope may provide valuable information and alert the surgeon as to a possibly unfavourable course.

\section{Sudden mydriasis}

In those cases of keratoconus where the air bubble injected at the end of the procedure happens to escape from the eye-an accident which takes place occasionally even when the sutures have been applied accurately and a precise fit has been achieved -the anterior chamber may be shallow at the first dressing and remain so for a day or two. Likewise, resorption of air without the formation of an equivalent amount of aqueous will result in a partially flat chamber. In patients submitted to penetrating keratoplasty for conditions other than keratoconus the anterior chamber may have been of little depth before surgery, particularly after the age of 50, in which case it will tend to become still shallower postoperatively owing to the flattening effect which the operation frequently exerts on the recipient cornea. This effect may become apparent only after the air which has been injected is resorbed.

If the pupil remains small, nothing untoward will happen. If, on the other hand, a sudden mydriasis develops, for example after the instillation of atropine, the ciliary part of the iris will come dangerously close to the posterior surface of the host cornea and at some point make contact with it (Fig. 1). This cornea-iris touch, which does not seem to have attracted much attention, is nothing short of disastrous, as it will perpetuate the mydriasis and trigger the development of a diffuse atrophy of the mesenchymal iris layers. That this is not caused by pupillary block is shown by the fact that after cataract extraction, where blocking of the pupillary opening through herniation of the hyaloid-contained vitreous is not rare and may be unrelieved for many hours, the above features are never observed as part of the regular picture. Pupillary dilatation, which eases pupillary block, is precisely what in most cases launches the whole cycle of events (UrretsZavalía, 1965).

Consequently, such contact should be broken

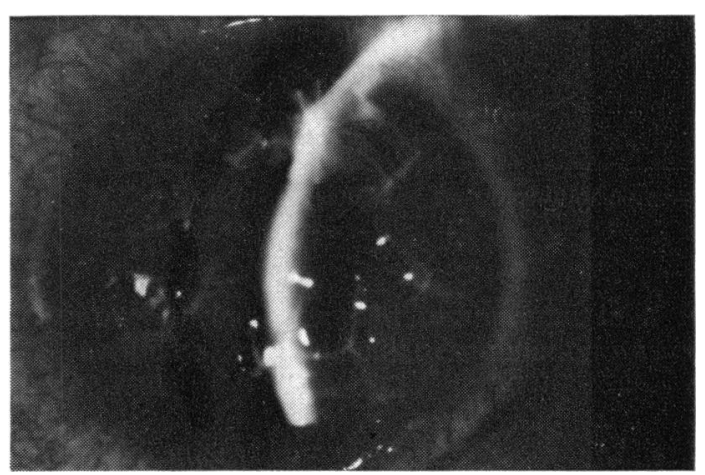

Fig. 1 Partial penetrating keratoplasty for keratoconus. Large, irregular mydriasis appeared after marginal corneairis touch developed between the $120^{\prime}$ clock and the 3 o'clock meridians 
without delay. This is done in the operating room with a long cyclodialysis spatula, by means of the technique devised by Castroviejo for the release of anterior synechiae. To avoid a recurrence of the adherence the anterior chamber is reformed with air or saline. As soon as the contact is broken the pupil will start to contract, until it regains its normal dimensions in a matter of days. Its reactions may remain sluggish, however, for a considerable period of time.

In the absence of any such peripheral touch between cornea and iris, the syndrome of acute mydriasis with iris atrophy may also develop (Fig. 2). It is as a rule irreversible and was first described by this writer (Urrets-Zavalía, 1963). The one thing that can be said in its favour is that in almost every case where it appears the graft will retain its transparency. It is as if the immunological phenomena which are responsible for graft rejection were mediated chiefly by cells contained in the affected iris layers, not by the circulating lymphocytes.

Perlon continuous sutures tend almost universally to be left in place for several months. In fair-skinned patients this entails no drawbacks; but in heavily pigmented patients in whom cicatricial tissue is often exuberant owing to hyperplastic collagen formation, the sutures may act as an irritant and result in the development of scattered marginal opacities with an irregular surface (Fig. 3). While these will not interfere directly with vision, they may hinder the

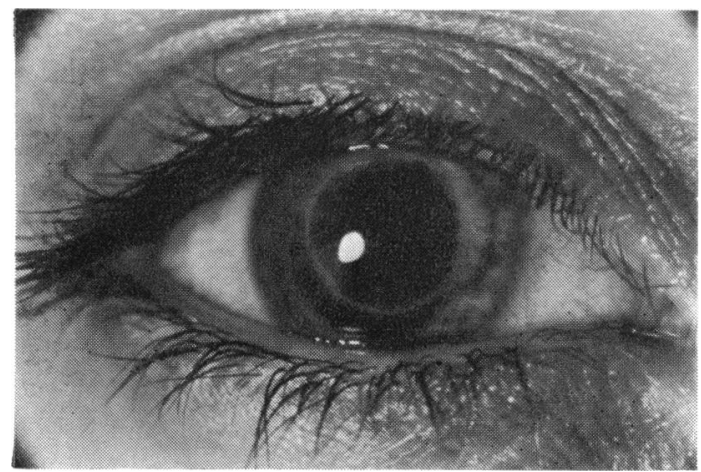

Fig. 2 Partial penetrating keratoplasty for keratoconus. Widely dilated, rigid pupil with iris atrophy which appeared spontaneously in early postoperative course

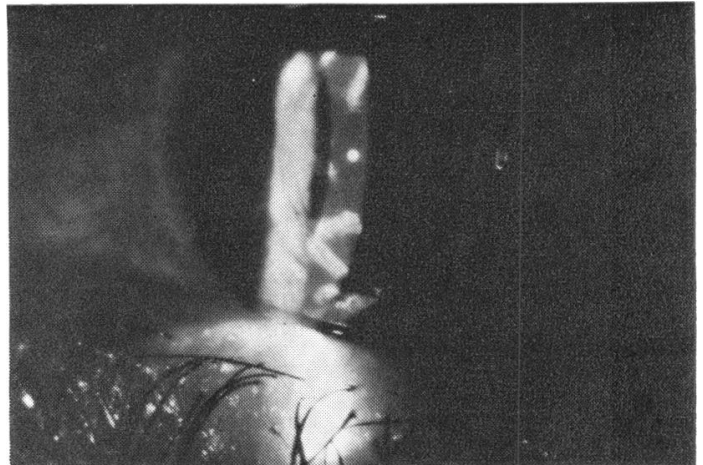

Fig. 3 Partial penetrating keratoplasty. Marginal minikeloid formation which developed along monofilament perlon suture tracts in brown-eyed individual

correction of the final astigmatic error whenever the graft is $7.5 \mathrm{~mm}$ in diameter or less. Keratometric readings become difficult to take, or cannot be taken at all, for the mires appear grossly distorted as they are reflected by the periphery of the graft. Hence, the patient will have to be fitted with a contact lens. This minikeloid formation may be avoided if the eye is closely watched with the slit-lamp, and the suture removed as soon as the scar seems to lose a purely lineal character.

Exceptionally, infection may develop along the track of one or several of the interrupted sutures, not along the track of the continuous ones, which are promptly covered by epithelium. If it is recognised in time and the offending stitches are removed, all will be set to rights with a few instillations of antibiotic drops. If the presence of the infected stitch is overlooked, not only will the infection persist but it will give rise to neovascularisation, which will reach the graft in no time.

\section{References}

Sánchez, J., and Polack, F. M. (1974). Investigative Ophthalmology, 13, 17.

Barraquer, J., and Rutllán, J. (1969). Cirugía del Segmento Anterior del Ojo, vol. 2, p. 371. Barcelona.

Urrets-Zavalia, A. (1963). American Journal of Ophthalmology, 56, 257.

Urrets-Zavalía, A. (1965). In The Cornea World Congress, eds. J. H. King, Jr, and J. W. McTigue, p. 700. Washington, Butterworths. 